\title{
X-RAY PHOTOEMISSION STUDY OF SATELLITE STRUCTURE ACCOMPANYING CORE IONIZATION FROM COORDINATED NITROGEN
}

\author{
Hans-Joachim FREUND. Harald PULM. Bernhard DICK \\ Lehrstuhl für Theoretische Chemie der Universitāt zu Köln. Greinstrasse 4. 5000 Cologne 71 . W'esr Giermamy. \\ and \\ Ralph LANGE

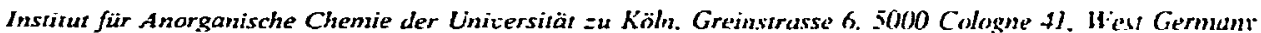

Received 25 January 1983; in final form $6 \mathrm{July} 1983$

\begin{abstract}
We compare the core ionization of free and adsorbed $\mathrm{N}_{2}$ to $\mathrm{N}_{2}$ bound head-on (with a linear $\mathrm{N}_{2}-$ metal hond) to a metal atom in a mononuclear organometallic compound, namely $h^{5}$-dicarbonyl-cyclopentadien! l-dinitrogen-nunganese(l). A comprebensive assignment is given for the full spectrum including main lines and satellite lines on the busis of numerical calculations. The conclusions are compared to those earlier derived for carbon-mononide complexes and adsorbares. The importance of doubly excited configurations for a correct description of satellite excitation energies is described.
\end{abstract}

\section{Introduction}

In 1978 Fuggle and co-workers [1.2] found intense satellite structure (called shake-up) accompanying the core ionization of $\mathrm{N}_{2}$ and $\mathrm{CO}$ adsorbates. An explanation of these results was provided by Gunnarsson and Schönhammer [3] in terms of a model hamiltonian treatment. These authors showed that the ionization probability known from the free molecule is redistributed upon adsorption of the molecule due to screening of the core hole on the molecule by metal electrons. The shape of the observed spectral function, e.g. energy distribution of ionization probabilities, was thought to be determined by the particular shape of the density of states of the metal substrate. Subsequently it was shown by comparison between molecular coordination compounds and adsorbates [4.5] that a few metal atoms were already sufficient to reproduce a core- and valence-hole spectral function that is virtually identical to the one observed in an adsorbate. These findings indicated that core- and valence-ionization spectra can be interpreted in terms of the well-known "surface-molecule approach" [6]. These comparisons were primarily carried through for coordinated $\mathrm{CO}$ systems with relatively strong bonding. In these cases the spectrum did not depend very remarkably on the metal involved in the bond. It was questioned [7]. however. whether this approach is applicable to systems with weak substrate-adsorbate interaction. Very recently cluster calculations using the $\mathrm{X} \alpha \mathrm{MS}$ procedure [8] showed that the proposed extension of the surface-molecule approach does work for the case of weakly chemisorbed $\mathrm{CO}$ on $\mathrm{Cu}$. Unfortunately weakly bonded $\mathrm{CO}$ complexes are not available. Other examples with weak moleculesubstrate interaction are $\mathbf{N}_{2}$ adsorbates which have been studied experimentally [2.7-13] and theoretically $[14-18]$ in the core- and valence-electron region on various metal substrates.

With $\mathrm{N}_{2}$ coordinated systems we are in the position to compare a weakly chemisorbed species in an adsorbate to a weakly bound molecule in a real complex. Therefore it was decided to study. the photoemission from $N_{2}$ adsorbates and $N_{2}$

0301-0104/83/0000-0000/\$03.00 @1983 North-Holland 
complexes Compared to $\mathrm{CO}$ systems the $\mathrm{N}_{2}$ systems are less stable by $\approx 20 \mathrm{kcal} / \mathrm{mol}$ per molecule-metal bond, both in complexes and adsorbates. To be specific, for $\mathrm{CO}$ and $\mathrm{N}_{2}$ adsorption on a $\mathrm{Ni}(110)$ surface the bond enthalpies differ by $84.4 \mathrm{~kJ} / \mathrm{mol}[19.20]$, and the reaction enthalpy for ligand exchange in, e.g $\mathrm{Ru}\left(\mathrm{NH}_{3}\right)_{5} \mathrm{~N}_{2}^{2+}$ is lower by $74.4 \mathrm{~kJ} / \mathrm{mol}[21,22]$ than in $\mathrm{Ru}\left(\mathrm{NH}_{3}\right)_{5} \mathrm{CO}^{2+} \ddagger$.

We report here on the first detalled experimental and theoretical comparison between the $\mathrm{N} l \mathrm{~s}$ core spectrum of a mitrogen complex namely $h^{5}$-dicarbonyl-cyclopentadienyl-dınıtrogen-manganese (I) with free nitrogen [23] on the one hand. and $\mathrm{N}_{2}$-transition-metal adsorbates $[8,24]$ on the other. We have chosen this complex for three reasons: (1) there are no other nitrogen atoms on other ligands present in the complex, (11) there is only one nitrogen molecule in the complex so that there exists no ambiguity for assignment of the spectrum and (111) the equivalent $\mathrm{CO}$ complex is known [21] The present companson leads to an assignment of the full range of satellites accompanying $\mathrm{N} l s$ core ionization and to the distinction between those excitations known from the free molecule and those characteristic of the bound molecule. Also, our study indicates that a surface-molecule description indeed is applicable to a weak chemisorptive bond as represented by a $\mathrm{N}_{2}$-metal adsorbate, as far as the interpretation of the photoemission spectra is concerned

In this paper. we first describe the experimental procedure to generate the spectra Then we proceed with a short description of the theoretical methods used to carry through the calculations of the core-hole spectral function. In section 3, we describe the qualitative ideas on which our interpretation is based. Section 4 contains a detailed discussion of the experimental and theoretical results in the context of known results from the literature. The last part contans a synopsis

* The absolute binding energies are $1509 \mathrm{~kJ} / \mathrm{mol}$ for ligand exchange in the CO complex [22] and $125.4 \mathrm{~kJ} / \mathrm{mol} \mathrm{in}$ the CO adsorbate [19], $765 \mathrm{~kJ} / \mathrm{mol}$ for ligand exchange in the $\mathbf{N}_{2}$ complex [21] and $410 \mathrm{~kJ} / \mathrm{mol}$ in the $\mathbf{N}_{2}$ adsorbate [20]

\section{Technical details}

\section{Experimental procedure}

The photoelectron spectra were recorded using a modified Leybold-Heraeus LHS-10 spectrometer in a d $E / E=$ constant [25] mode The samples were prepared by sublimatıng the complex onto a lıquid-nitrogen-cooled metal ( $\mathrm{Fe}, \mathrm{Cu}$. $\mathrm{Ag}$. $\mathrm{Au}$ ) substrate. The samples were kept at liquid-nitrogen temperature during data acquisition. This was necessary to prevent the complex from evaporation. The energy scans were $50 \mathrm{eV}$ wide and were covered by 256 channels using a dwell time of $2 \mathrm{~s} /$ channel. The spectrum shown in fig. 1 was recorded by averaging the data of four scans and subtracting the signal from the metal substrate. We have chosen the highest possible intensity achrevable with the instrument at the cost of high resolution. The resolution was kept at $1.7 \mathrm{eV}$ (fwhm). In this way we were able to get a reasonable signal-to-noise ratio at binding energies further below the main line. The spectra presented have been reproduced using several different metallic substrates in order to rule out possible contributions to the spectrum from electrons of the metal substrates The effect of disintegration of the complex on the spectrum was studied by heating a sample before the spectrum was taken and by irradiating the sample for $30 \mathrm{~min}$ before data acquisition. Using this procedure we can rule out the possibility of contributions from disintegration products to the spectrum shown in fig. 1 .

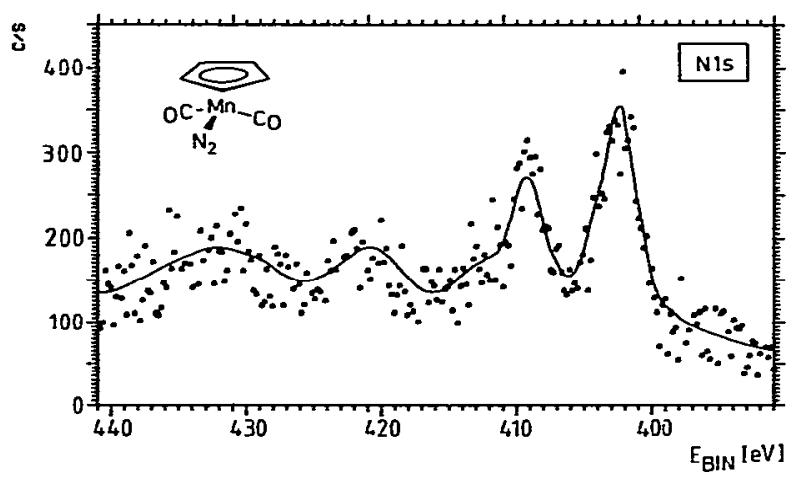

Fig 1 Nls spectrum of $\left(\mathrm{C}_{5} \mathrm{H}_{5}\right)(\mathrm{CO})_{2} \mathrm{MnN}_{2}$ recorded with MgKa radiation 


\section{Computational procedure}

Calculations were done using a semi-empirical CNDO LCAO SCF algorithm recently pardmeterized to mimic ab initio single-particle properties of transition-metal compounds [26.27]. The result obtained by this method is then augmented by a configuration-interaction calculation including doubly excited configurations [28]. In order to describe the core-ionized species we employed the equivalent-core method [29.30] We substitute the atom with nuclear charge $Z$ which is to be ionized by the atom with the next higher nuclear charge $(Z+1)$ and calculate the system with unaltered number of valence electrons. This procedure allows us to calculate the motion of the valence electrons in an effectively changed core potential and models the change in potential upon core ionization. The excited core-1onized states were calculated by a configuration-interaction treatment including single and double excitations [28] In all ionic-state calculations we selected 300 configurations with lowest excitation energy out of 5000 created configurations of proper symmetry. Relative intensities were calculated by projection of the wavefunction of the correlated ion states onto the wavefunctions of the neutral system. according to the sudden approximation [31].

$I_{\text {rel }}^{\prime} \propto\left|\left\langle\Psi_{\text {relaxed }}^{\text {ion }} \mid \Psi_{\text {frozen }}^{\text {ion }}\right\rangle\right|^{2}$.

Within this approximation a sum rule holds stating that the first moment of the spectral function is equal to the Hartree-Fock eigenenergy [32]:

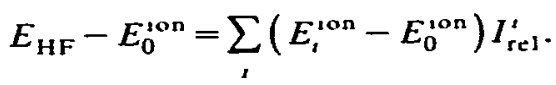

Since the states involved are linear combinations of determunants created from the set of self-consistently determined orbitals of the neutral $\phi_{,}$and the core-ionized $X$, system, we can write the intensity as [33]:

$$
\begin{aligned}
& I_{r=1} \propto\left|\left\langle\sum_{t} c_{l}\left\|x_{h}^{2}\right\| \mid \sum_{J} d_{j}\left\|\phi_{1}^{0}\right\|\right\rangle\right|^{2} . \\
& I_{\mathrm{r}=1} \propto\left|\sum_{t} \sum_{J} c_{l} d_{j}\left\langle\left\|x_{h}^{2}\right\|\|\| \phi_{1}^{0} \|\right\rangle\right|^{2}
\end{aligned}
$$

$I_{r a 1} \propto\left|\sum_{1} \sum_{j} c_{2} d, \|\left\langle x_{k}^{\prime} \mid \delta_{1}^{0}\right\rangle\right|^{2}$.

which is easily programable. We melude in the intensity calculations only thone doublet states that result from spin combinations represented by a singlet excitation in the valence shell The spectral function calculated in this $w$ al can be used to evaluate the sum rule. This in turn allows us to calculate relasation energies since this quantity is given as the difference hetween the Hartree-Foch eigenenergy and the true ionzation potential.

\section{Qualitative considerations}

For illustrative purposes let us consider the most simple case of a dixtomic molecule (AB) interacting with a single metal atom (Me). The orbitals of the neutral sistem resultung from the metal $\mathrm{d} \pi$ (hereafter called $\mathrm{d}-$ ) molecule -* (hereafter called ${ }^{*}$ ) interaction (--acceptor interaction) and from the molecule $\sigma$ (hereafter called o) metal do/s (hereafter edlled do) interaction ( $\sigma$-donor interaction) are schematically show $n$ in f1g. 2a [5] A non-interactung molecular - orbital (heredfter called $\pi$ ) is also indicated. The orbitals appear to be localized predommantly on the two parent fragments With respect to the uncoordinated free molecule the unoccupied ${ }^{-*}$ orbital becomes partly populated. While the occupied $\sigma$ orbital becomes partly depopulated. If we now introduce a core hole on the molecule. e.g. on the center close to the metal. the Coulomb interaction stabilizes the unoccupied -* and occupied $\sigma$ and levels on the molecule with respect to the metal levels. This causes the mixing between molecular and metal levels to change. The main result is an increased mixing between the $\pi^{*}$ and the $\mathrm{d}-$ orbitals and a decreased mixing between the $a$ and the do orbitals This is indicated in $f_{1} g$ 2b bs changing the weight of the LCAO coefficients on the parent fragments. If we now project the wlence orbitals of the core-1onized species onto those of the free molecule we find the $\pi^{*}$ orbitals of the AB molecule with larger population than in the neutral system. Population of the $\sigma$ orbital increases with respect to the neutral coordinated 


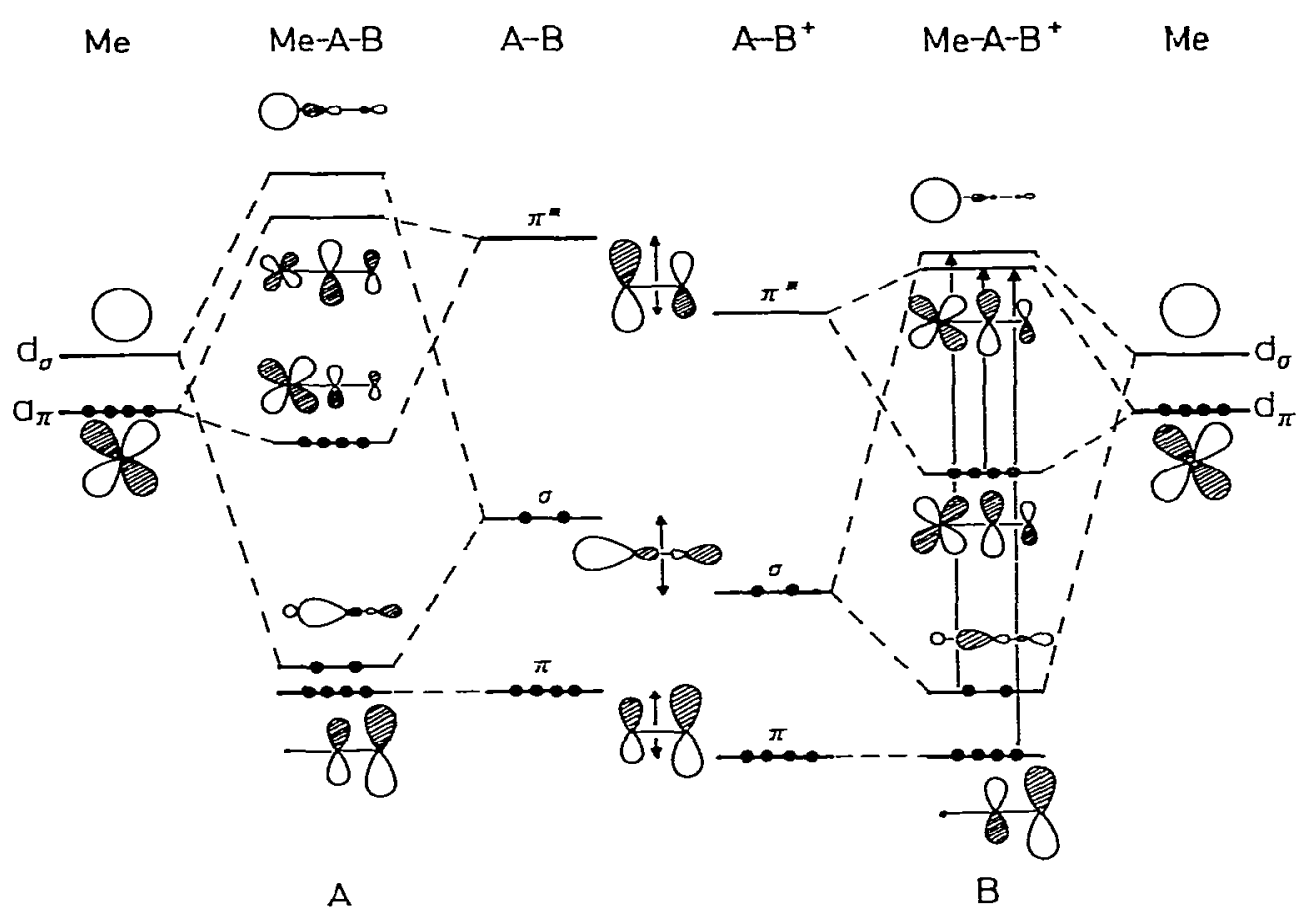

Fig 2 Schematic representation of the interaction of the valence orbitals of a metal atom (Me) with a diatomic molecule (AB) in the neutral (A) and the core-ionized (B) system Arrous in B indicate possible shake-up excitations

system This redistribution of electrons in the valence region induced by the ionization process (fig. 2b) leads to an accumulation of electronic charge on the molecular moiety and thus to a screening of the core hole. The details of this screening mechanism determine the observed spectral function, in particular the intensity distribution.

We use as an example to show thus the one-electron states depicted in figs. $2 a$ and $2 b$. In order to calculate the intensity distribution we project the occupied orbitals of fig. $2 b$ onto those of fig. $2 a$ according to eq. (1). Clearly the overlap is not unity since the shape of the orbitals has changed upon ionization. If we now excite an electron into the unoccupied orbitals, e g. $\pi^{*}$, out of $\pi$ we create a state that has a finite overlap amplitude with the occupied orbitals of fig. $2 \mathrm{a}$ and therefore has a finite ionization amplitude. The absolute magnıtude of this overlap amplitude is sensitive to the size and sign of the LCAO coefficients of the wavefunctions. These LCAO coefficients in turn depend on the interaction between molecule and metal. We have shown [5] that for weaker metal-molecule interaction as compared to carbonyl complexes the overlap amplitude of frozen and equivalent-core-ion ground state, which determines the shake-up intensity, should increase. At the same time the energy separation between satellite and main line should decrease since the splitting between bonding and antibonding contr1butions gets smaller. The latter statement however, holds only if the nature of the excited state stays the same as the metal-molecule interaction vanes Usually it is assumed that the excited state can be described as a single excitation. However, if the splitting becomes small and the overlap matrix element for a certain singly excited state becomes rather large then the overlap matrix element for a double excitation involving the same orbitals but a larger excitation energy is likely to get large too. The singly and doubly excited states mix, and consequently the intensity may be partly 
transferred into the doubly excited state. After this type of configuration mixing has been included, the intensity of the shake-up spectrum may peak at a higher excitation energy than expected without this additional interaction. We conclude therefore. that, even though the metal-molecule bond strength gets smaller, the observed shake-up exc1tation energy may increase. The intensity of the excitation may increase as well.

\section{Results and discussion}

\section{Experimental results}

$\mathrm{N}_{2}$ on $\mathrm{N}_{1}$ [8.11] and $\mathrm{CO}$ on $\mathrm{Cu}$ [34] represent. as mentioned before, cases of rather weak chemisorptron as compared to $\mathrm{CO}$ adsorption on other transition metals. The model outlined in the previous section predicts rather intense satellite structure for these weakly chemisorbed systems in general, if the metal-molecule interaction determines the intensity of the satellite structure. For $\mathrm{N}_{2}$ adsorbates these satellites have indeed been observed [8.11]. It has been claimed that in those cases the complete spectral function and particularly the fine structure of the main line is mainly connected with the particular shape of the density of states of the substrate and that the structure in the main line is not due to the inequivalency of the nitrogen atoms [7]. This argument was based on the slight differences observed in the fine structure of the leading peak when adsorption on various substrates was compared [7].

For $\mathrm{N}_{2}$ complexes there exist only very fragmentary data on $\mathrm{N}$ ls satellites [35], while there is a full body of measurements on N1s main lines [35-39], namely to demonstrate the inequivalency of the two nitrogen atoms after coordination to a metal atom. Leigh et al. [35] were the first to report on the split $\mathrm{N}$ ls photoemission signal from rhenium- $\mathrm{N}_{2}$ complexes. Therr spectra do show satellite structure on the high-binding-energy side of the Nis main line but these authors did not discuss the extra structure. Our spectrum (see fig. 1) shows intense satellite structure on the highbinding-energy side of the Nls main line, with an energy separation of 7-8 eV. Binder and Sellmann

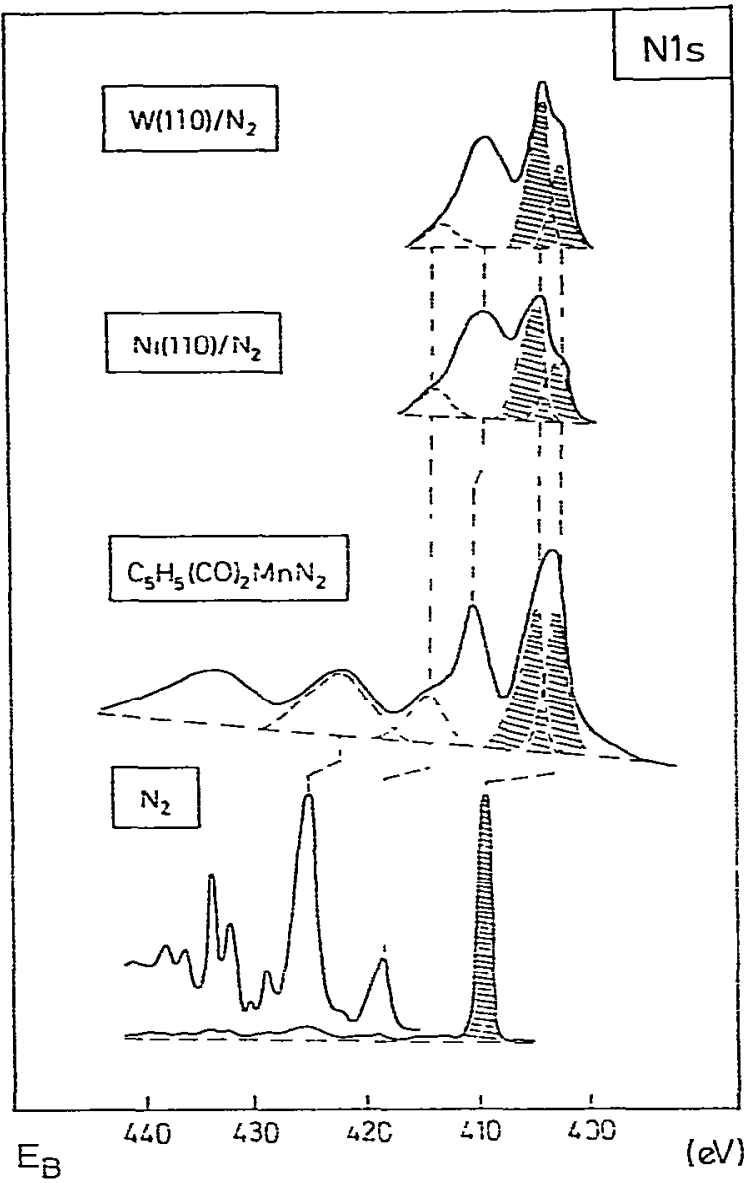

Fig 3 Comparison of $\lambda l s$ spectra of free $N_{2}$ [23]. $\left(\mathrm{C}_{5} \mathrm{H}_{1}\right)(\mathrm{CO})_{2} \mathrm{MnN}_{2}$ and the adsorbate systems $\mathrm{N}_{1}(110) / \mathrm{N}=$ [9] and $W(110) / N_{2}[24]$ The bunding energies of the leading peah in the adsorbate ststems hate been aligned with those of the complex

[36] have studied the identical complex but did not investıgate the satellite region. They studied the main line with higher resolution and found a split main line with a peak separation of $\approx 2 \mathrm{eV}$. It should be noted that the spectrum of Binder and Sellmann [36] very well fits underneath our main line.

Fig. 3 compares the spectra of the $\mathbf{N}_{2}$ complex with those of free $N_{2}$ [23] and $N_{2}$ adsorbates [9.24] taken from the literature. Fig 3 is intended to use the known assignment of the satellite structure of free $\mathrm{N}_{2}$ [40] to establish a comprehensive assign- 
ment for the coordınated systems.

The two satellites at lowest excitation energy in the free molecule have been assigned to be due to the two doublet states resulting from the $1 \pi_{\mathrm{u}}-1 \pi_{\mathrm{g}}^{*}$ excitation in the presence of the core hole [40]. It is known that the states originating from a $1 \pi_{u}-1 \pi_{\mathrm{b}}^{*}$ excitation are split by $\approx 7 \mathrm{eV}$ as a result of configuration interaction $[40]^{\ddagger}$. The intensity of the shake-up satellites caused by these states is rather small The intensity of the satellite above $16 \mathrm{eV}$ excitation energy is by a factor of 3 larger than that of the one at $\approx 10 \mathrm{eV}$. This latter satellite borrows its intensity through secondary spin coupling [5]. The satellites as well as the main line shift towards lower binding energy upon coordination (see fig. 3) since in the presence of the hole the excited electrons feel the changed potentual caused by extramolecular screening by metal electrons The various peaks are shifted towards lower binding energies by slightly different amounts due to the fact that the final state $\left(\pi^{*}\right)$ of the excitation is not identical to the free molecule. In particular the $1 \pi_{u}-1 \pi_{\varepsilon}^{*}$ satellite at small excitation energy is shifted close to the region of very intense satellites. Which inhibits us to locate this peak definitely. In addition to the peaks that are due to excitations on the adsorbed molecule the most intense satellite occurs at $\approx 7 \mathrm{eV}$ below the main line We assign this peak to excitations between those orbitals resulting from the coupling between $\mathrm{N}_{2}$ and metal levels as discussed in section 3 . The electronic excitations giving rise to this satellite peak take place into the same level $\left(\pi^{*}\right)$ as in the case of the local intramolecular $\mathbf{N}_{2}$ satellites, but start from an orbital with large metal character as opposed to the high $\mathrm{N}_{2}$ character for the starting level of the local $N_{2}$ excitation (see $f_{1} g$ 2b). This will be discussed further below. The structure at $\approx 29 \mathrm{eV}$ excitation energy has not been discussed so far. It is not clear how to assign it. Whether it is due to shake-off structures (observed in this energy range for $\mathrm{CO}$ coordinated systems) could be decided on the basis of the Auger N-KLL spectrum of the complex [4]. Studies in this direction are in progress.

* See also ref [41] for the splutung of the $\pi-\pi^{*}$ excitation in free $\mathrm{N}_{2}$
This qualitative discussion. presented so far. shows that a comprehensive, unique interpretation of the satellite structure of compounds and adsorbates can indeed be found on the basis of a surface-molecule concept. The excitations on the adsorbed molecule can be identified in comparison with the free molecule. Intermolecular excitations are found. the intensities of which are in accord with qualitative predictions.

\subsection{Theoretical results and comparison to experiment}

In order to lend additional theoretical support to our qualitative interpretation we present the results of a configuration-interaction study on the entire complex to describe the two possible Nls core-hole ionizations of the complex as well as the electronic structure of the neutral system Fig 4 contains the charge distribution calculated fo: the

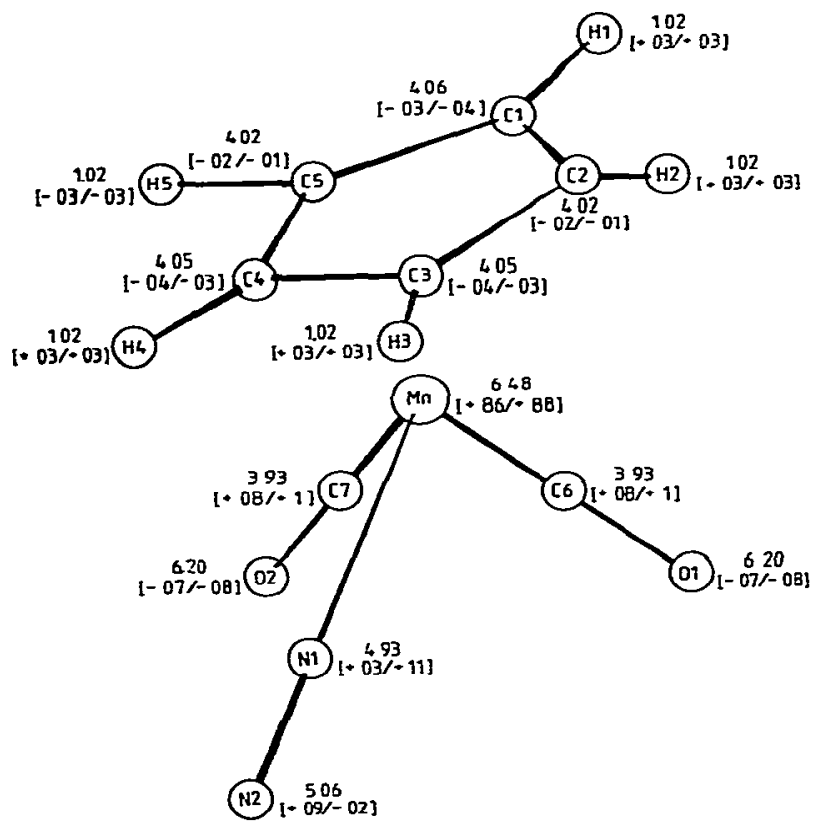

Fig 4. Atomic electron densities for the neutral molecule and charge densities for the ions The first value in brackets is the charge density upon core ionization of the metal adjacent nitrogen (N1), the second is the charge density upon core ionization of the nutrogen further separated from the metal (N2) 


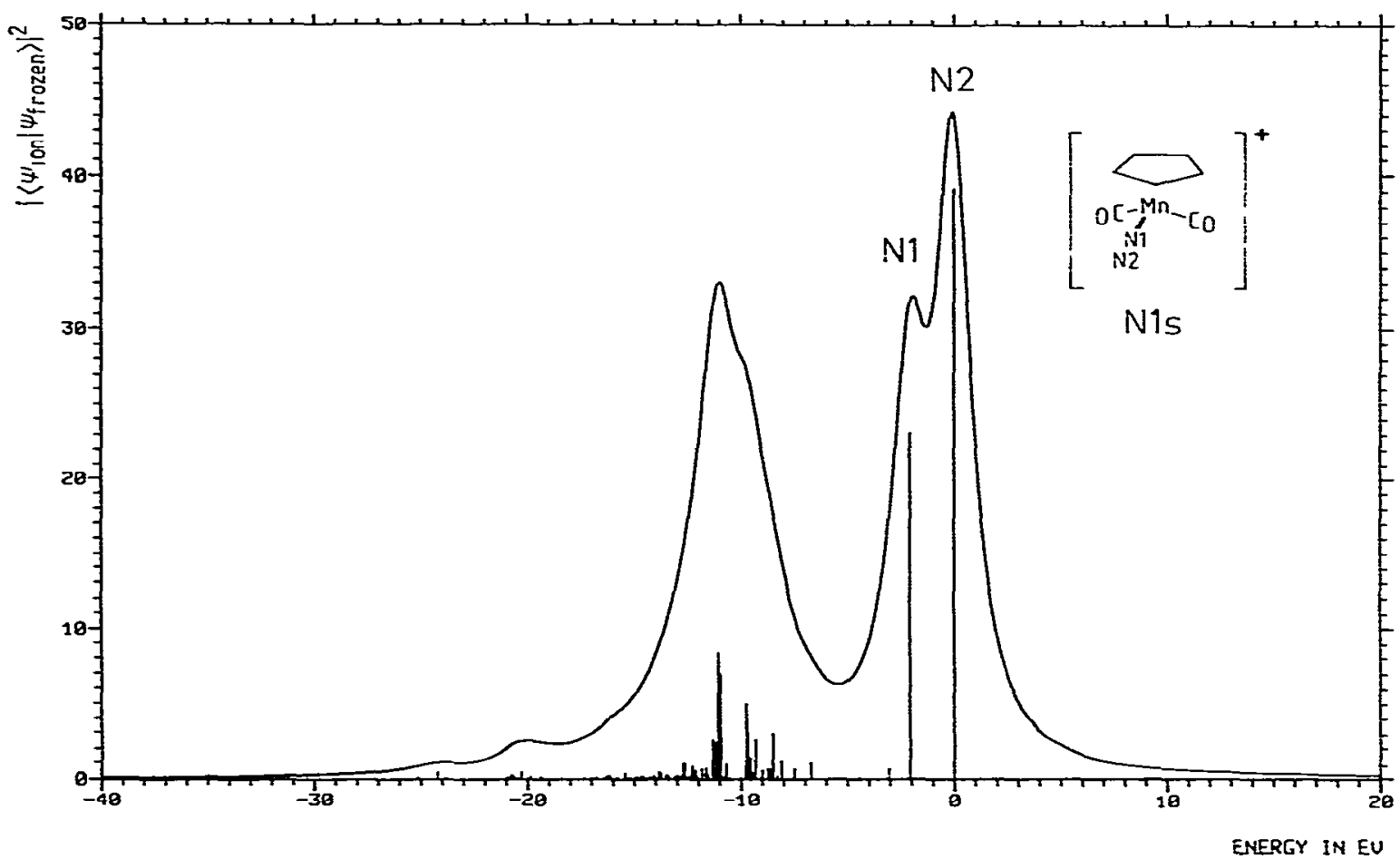

Fig 5 Calculated Nis spectrum of $\left(\mathrm{C}_{5} \mathrm{H}_{5}\right)(\mathrm{CO})_{2} \mathrm{Mn} \mathrm{V}_{2}$ - Energies are given relatue to the line at lowest hinding energi Spectrum convoluted by a lorentzian of $\mathrm{fwhm} 17 \mathrm{eV}$

ground state of the neutral system and for the two equivalent-core states. The important result for the further discussion is the charge distribution on the nitrogen moiety for the neutral system. We find the nitrogen atom separated from the metal atom negatively charged, while the metal adjacent nitrogen carries a positive charge. Using this charge distribution we calculate a relative chemical shift for the two nitrogen atoms of $2.46 \mathrm{eV}$ with the metal adjacent nitrogen at higher binding energy. We used the ground-state potential model as discussed by Gelius [42] and employ the optımal parameter for nitrogen [30]. For comparison with experimentally observed chemical shifts differentral relaxation energies have to be included. In order to do this according to the procedure outlined in section 3 the complete spectral function of each core hole has to be known. Fig. 5 shows the superimposed spectral functions of the two core holes. Fig. 6 shows the separate contributions. The sum rule [eq. (2)] yelds a relaxation energy by 0.4 $\mathrm{eV}$ larger for the metal adjacent mitrogen as compared to the second nitrogen which then decreases the relative chemical shift to $2.06 \mathrm{eV}$. keeping the sequence of the core ionizations as expected from ground-state charge distribution. The calculated chemical shift of $2.06 \mathrm{eV}$ compares fortuitously well with the experimental value of Binder and Sellmann [36].

The consequences of the ionization process for the electron distribution for both nitrogen atoms are shown in fig. 7 . In fig. 7 the density-difference maps in the plane indicated in the inserts of fig. 6 are plotted. The $z$ axis is a measure for the charge density. The difference is taken between the frozen ion state. described by the valence-electron wavefunction of the neutral system. and the equivalent-core-ion ground state. For both hole states a rather high electron density accumulates on the ionized atom to screen the core hole. The 

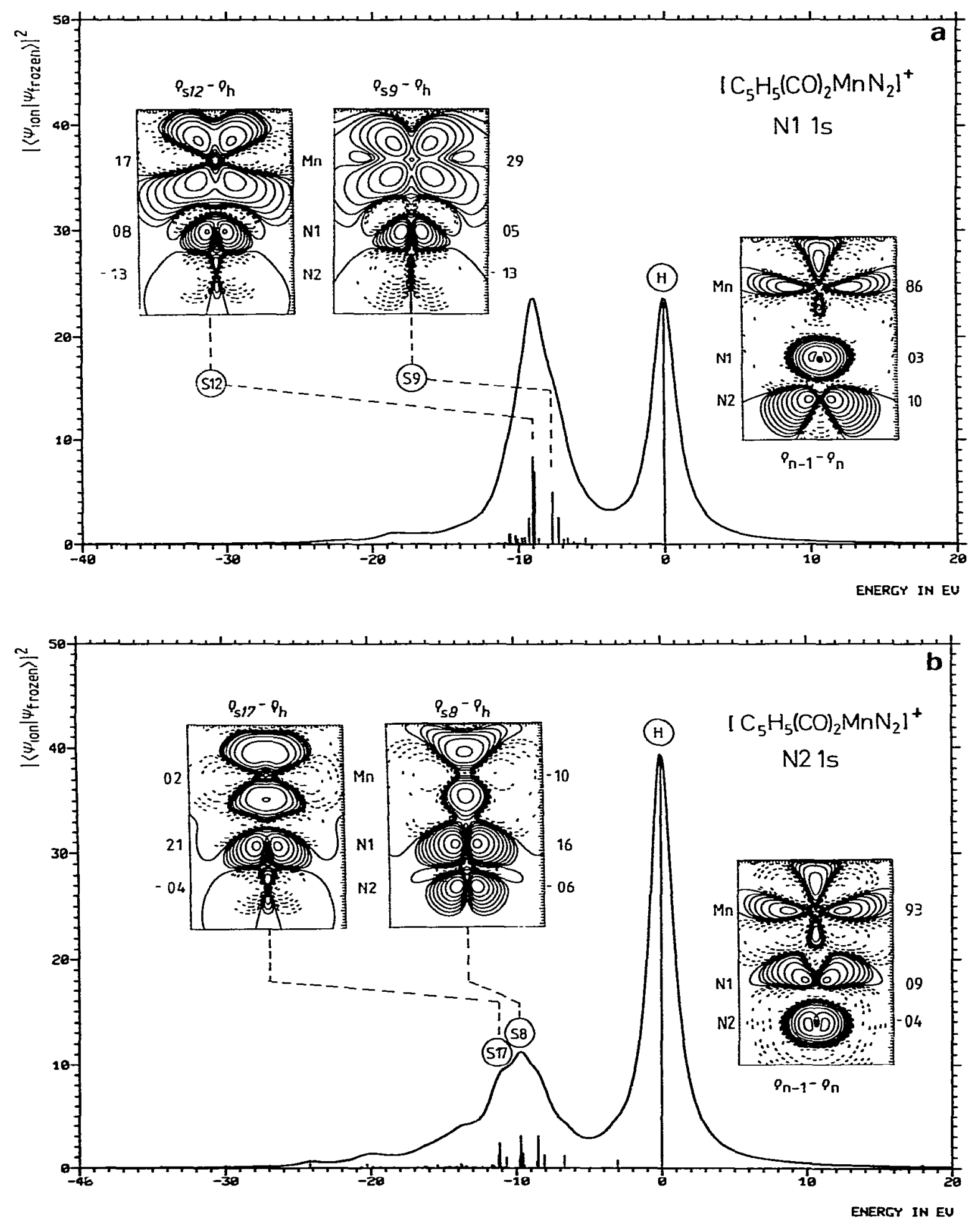

Fig 6. (a) Calculated $\mathrm{N} 1 \mathrm{~s}$ spectrum of the metal adjacent nitrogen of $\left(\mathrm{C}_{5} \mathrm{H}_{5}\right)(\mathrm{CO})_{2} \mathrm{MnN}_{2}$ For electron-density difference maps see text (b) Calculated Nis spectrum of the non-metal adjacent nitrogen of $\left(\mathrm{C}_{5} \mathrm{H}_{5}\right)(\mathrm{CO})_{2} \mathrm{MnN}_{2}$ For electron-density difference maps see text (The plotting plane is the same as in fig 7) 


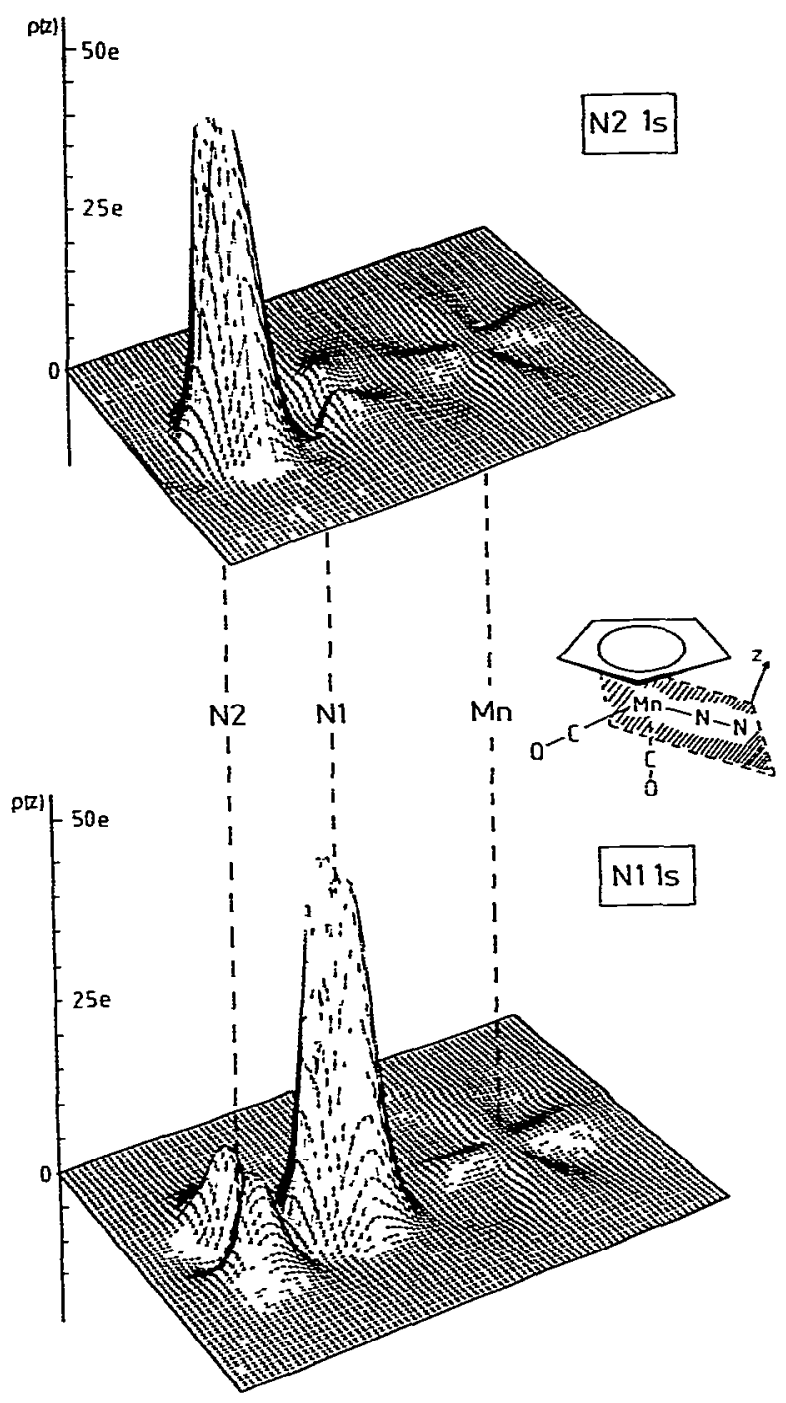

Fig 7. Three-dimensional plots of the pnmary hole states marked $\left(\rho_{\Lambda-1}-\rho_{\sqrt{3}}\right)$ in $\mathrm{fig}_{\mathrm{g}} 6 \mathrm{a}$ and $\mathrm{f}_{1 \mathrm{~g}} 6 \mathrm{~b}$ The plane chosen in the molecule is indicated

size of the peak indicates the localization of the screening charge. The absence of "negative" peaks of comparable size is another indication for this localization. It seems reasonable by inspection of fig. 7 that the relaxation energy is similar for the two core holes. The slightly larger relaxation energy of the metal adjacent nitrogen. however, has a rather strong influence on the relative intensities of the shake-up satellites for the two core holes (see $f_{1 g}$ 6) This is a quantitative documentation of the statement made in section 3 that the satellite intensity is very sensitive to the size of the LCAO coefficients of the wavefunctions. This will be discussed in detal further belou.

The calculated satellite excitation energies (see fig 5) as well as the overall reldure intensities are in qualitative agreement with the experiment of fig 1. Out of the 600 excited states used to calculate the spectral function of the two N Is core holes only 142 states get non-zero intensity. At this point it is interesting to investugate the influence of double excitations on satellite intensity and excitation energy. We have therefore attempted an equivalent calculation using only singly excited states. The result is presented for the two hole states in fig. 8 . The qualitative result is smular to the one found for the full calculamon. in particular as far as the trend in satellite intensities for the two different core holes is concerned. The relative satellite intensity. however. is much smaller in fig. 8 compared to fig. 6 . Also. the excitation energy is smaller as compared to an equiralent calculation of coordinated $\mathrm{CO}$ [43]. From this we learn that in the case of coordinated $N_{2}$ the doubly excited states play an important role to reproduce the observed rather large excitation energ: and the intense satellite structure. This result discussed already qualitatively in section 3 is quite in accord with chemical intuition. It is well hnown that a Hartree-Foch description fals to correctly describe the dissociation of a sistem. or the bonding in a weahly interacting system. In order to achieve the correct behaviour. correlation has to be taken into account For a closed-shell sistem the lowestorder contributions in a configuration-interaction expansion to describe the correlated $\mathrm{w}$ as efunction are doubly excited configurations [14] This is a redson why doubly excited states do not contribute as significantly in coordinated CO systems. where the metal-molecule bond is stronger [43].

Let us now investigate in detail the nature of the ion states involved in the most intense shake-up transitions. Using the electron density distribution inserted in figs. $6 \mathrm{a}$ and $6 \mathrm{~b}$ we can achieve this goal. We start with the N1s core hole on the metal adjacent nitrogen. On the right-hand side of $f_{1} g$ $6 \mathrm{~d}$ 

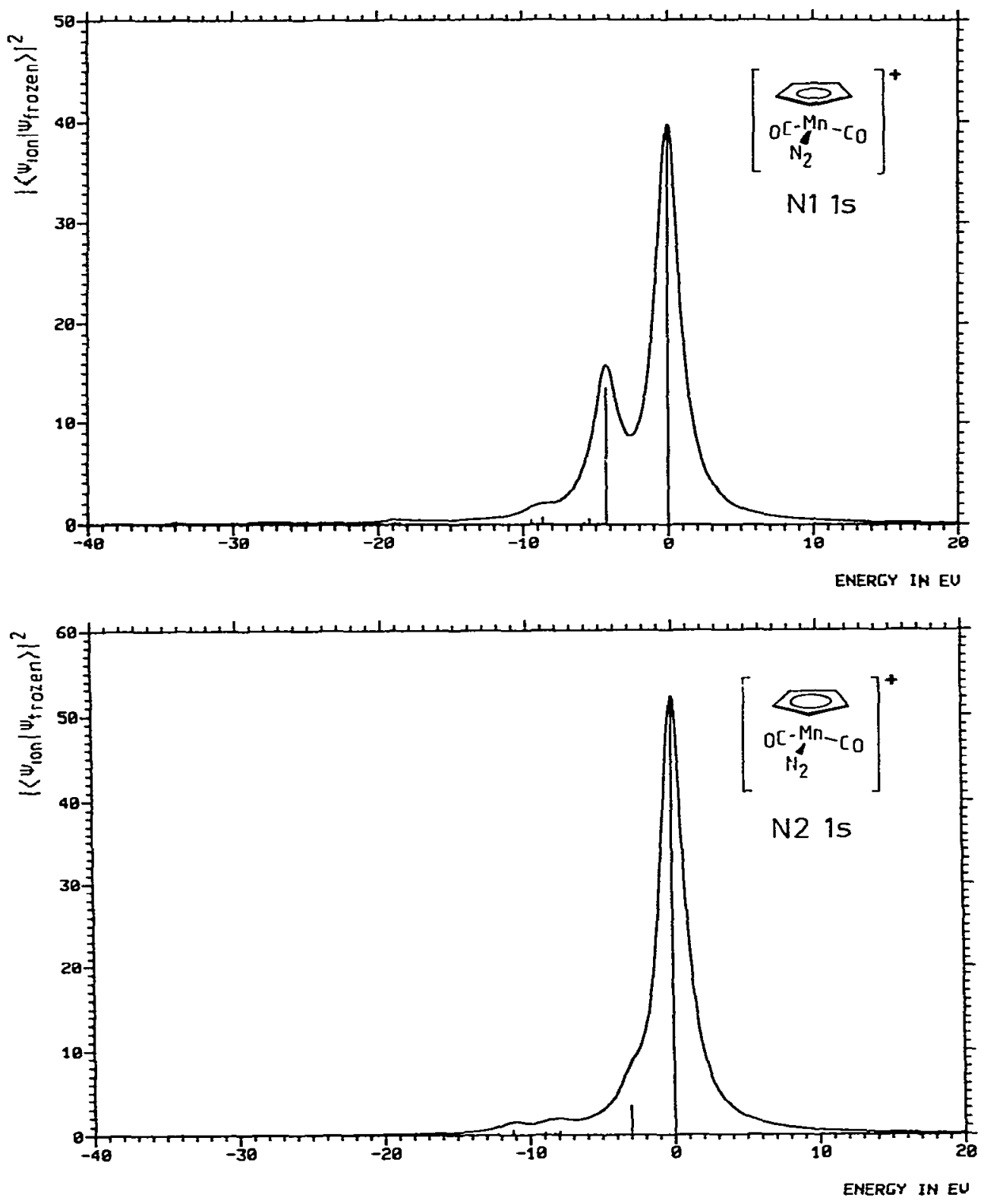

Fig 8 (a) Same as fig $6 a$ using only singly excited ion states (b) Same as fig 6b using only singly excited inn states

the electron-density difference map (see also fig 7 for a definition of the plotting plane) calculated from the frozen ion state, described by the valence-electron wavefunction of the neutral system, and the equivalent-core-ion ground state is plotted.
Solıd lines represent electron gain in the ion, while broken lines represent electron loss. The effect of core-hole creation on the valence-electron distribution is obvious: A rather high electron density accumulates on the 1onized atom to screen the core 
hole. As a consequence the valence-electron density is redistributed all over the system Within the plane plotted, the entire $\mathrm{N}_{2}$ moiety gains electrons. even the non-ionized nitrogen atom. while the metal atom looses electrons. The integrated total densities per atom are shown in $\mathrm{fig}_{1 \mathrm{~g}} 6$ for comparison. The net effect of the Nls ionization in the complex is a loss of $0.3 e$ relative to the neutral system, where the metal center already carries 052 $e$ positive charge. This loss is small compared to the electron-density loss calculated for $\mathrm{N}_{1}-\mathrm{CO}(0.9$ e) upon creation of a $\mathrm{Cls}$ hole. The larger charge transfer in the latter system is partly due to the higher electron density on the metal atom in the neutral $\mathrm{N}_{1}-\mathrm{CO}$ system In both cases. namely $\mathrm{Cls}$ ionization in $\mathrm{N}_{1}-\mathrm{CO}$ and Nls ionization in the manganese complex the metal atom carries an absolute charge of $\approx 0.9 e$ after ionization. This charge is transferred from the metal towards the coordinated molecule through a channel of $\pi$ symmetry We see from the plots of the hole states that simultaneously a small amount of charge is transferred back towards the metal through a o channel This is due to the increased coupling between unoccupied $\pi$ orbitals of the coordinated molecule but decreased coupling of the occupied $\sigma$ orbitals of the coordinated molecule to the metal after Ionization as pointed out in section 3 .

As examples for excited ion states we show the electron redistribution upon electron excitation for two states. To map out electron distributions the equivalent-core ground state has been subtracted from the excited states We have calculated the plots for those states that lead to large overlap with the frozen state. The largest contributing determinant, according to eq. (3). to the most intense satellites results from the HOMO-LUMO excitation in the core-ionized complex. The character of these orbitals is shown in $f_{1} g$ 9. The HOMO can be looked at as the bonding combindtion of the $1 \pi_{\mathrm{g}} \mathrm{N}_{2}$ orbital and a metal d orbital. while the LUMO is the antibonding combination of the same orbitals (see section 3 ). The distribution of atomic-orbital coefficients on the $\mathrm{N}_{2}$ motety constituting the molecular orbitals of the ion is very similar to the distribution in coordinated $\mathrm{CO}$ [43] The corresponding orbitals for the $\mathrm{Ni}-\mathrm{CO}$ system have been included in fig 9 for comparison

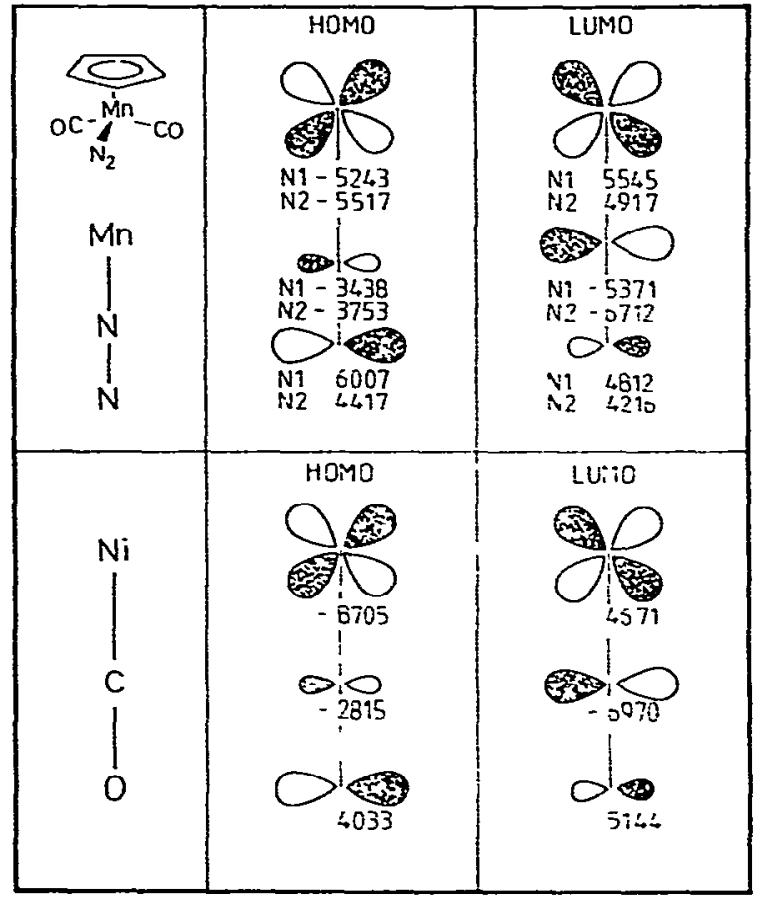

Fig 9 Schematic repreventation of the highes cocupled (HOMO) and Iowest unoceupted (LUNIO) molecular orbital of $\left(C_{2} H_{4}\right)(C O)_{2} M_{n} V_{2}$ in the reglon of the metal- $)_{2}$ bond The

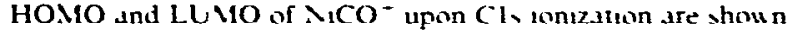
for comparioen [43]

[43] The unoccupled linear combination has a rather large coefficient on the ionized center. while the occupied linear combination has a larger coefficient on the non-1onized nutrogen atom. Therefore upon excitation charge is transferred tow ards the ionized center. It is interesting to note that through the excitation a considerable charge transfer comes from the non-ionized nitrogen. This is clearly seen in the first plot for one of the intense satellites. The second nitrogen looses $0.1 \mathrm{e}$. while the conized center gains $0.04 \mathrm{e}$ with respect to the equivalent-core ground state. A very similar situation is found for the other excited states below 16 $\mathrm{eV}$ excitation energy as exemplified. The state above $16 \mathrm{eV}$ excitation energy belongs to the $1 \pi_{u}-1 \pi_{s}^{*}$ excitation in free $N_{2}$. For comparison we shou in fig. 10 the calculated spectrum of free $N_{2}$. The intensity of this state is rather low, but its 


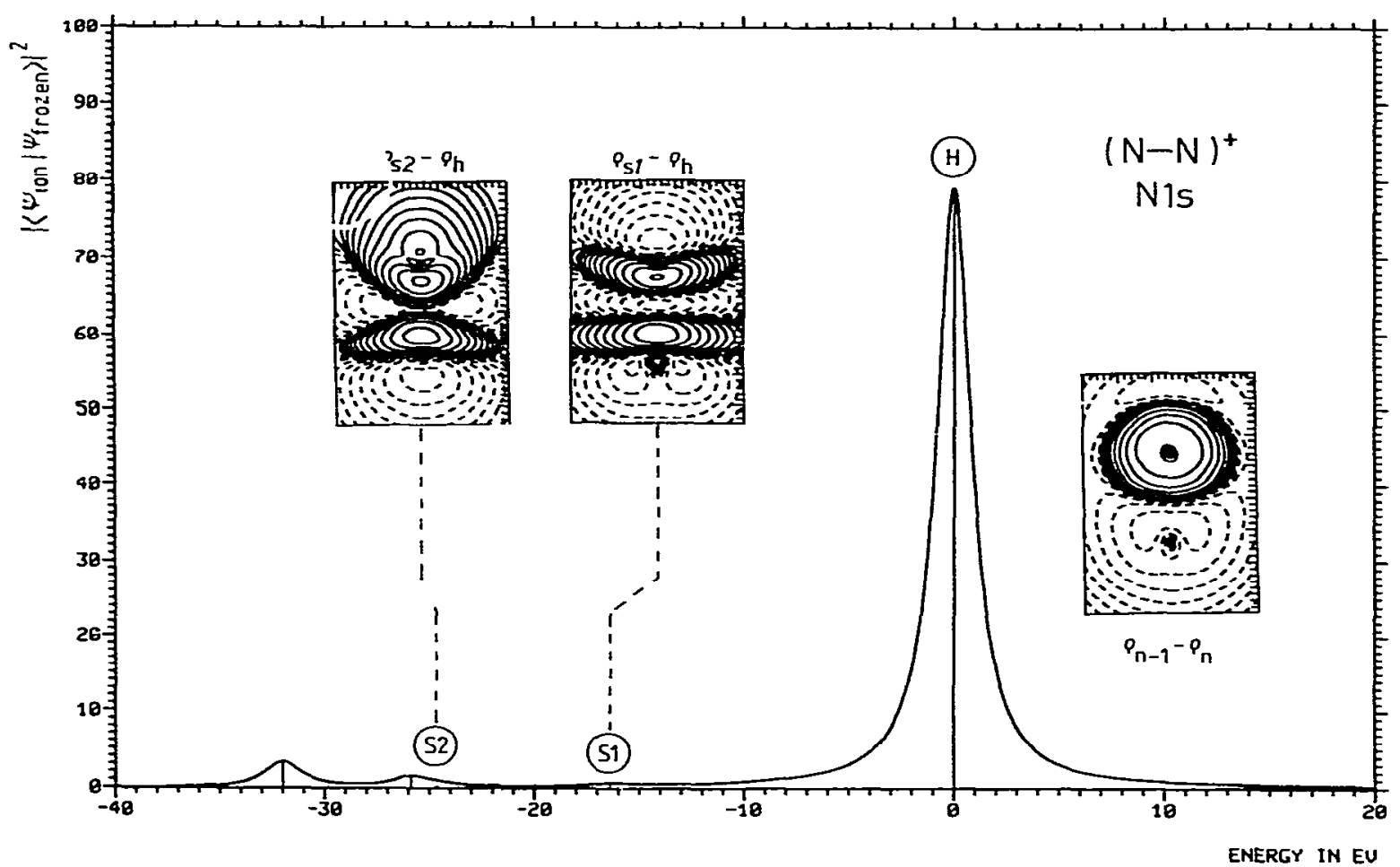

Fig 10 Calculated $N 1 s$ spectrum of free $\mathrm{N}_{2}$ using only those doublet spin combinations where the salence excitation can be described b) a singlet state [5]

energy is close to the observed peak

The discussion for the hole and excited hole states of the nitrogen further separated from the metal center proceeds very much in the same way The calculated (see fig $6 \mathrm{~b}$ ) reduced satellite intensity in the case of this $\mathrm{N}_{2}$ ionization can be traced back to the smaller overlap of determinants created from the HOMO-LUMO excitation and the frozen ion state As seen in fig. 9 the distribution of atomic coefficients in the HOMO and the LUMO for this ionization is slightly different from the one found for the ionization of the metal adjacent nitrogen. In particular, the coefficients on the $\mathrm{N}_{2}$ molety are more balanced in the occupied bonding combination, while the unoccupied antibonding combination has a large coefficient on the metal adjacent nitrogen. An excitation into this orbital therefore does not contribute to a screening of the hole on ionized nitrogen. Thus rationalizes the calculated differences in satellite intensity and re- laxation energy between the two inequivalent nitrogen core holes

\section{Conclusions}

From our study we draw the following conclusions.

(a) The photelectron spectrum of the Nls electrons of a coordinated nitrogen molecule bound within a funite complex exhibits strong satellite structure simlar to nitrogen adsorbates on transition-metal surfaces.

(b) The intensities of the satellites relative to the main lines for the coordinated nitrogen molecule are larger than for coordinated carbon monoxide. The excitation energy is larger than in the case of carbon monoxide.

(c) The experimental spectrum can be repro- 
duced by a configuration-interaction calculation using LCAO MO SCF wavefunctions on the basis of the equivalent-core approximation. The two inequivalent nitrogen atoms in the coordinated system lead to ion states that are energetically shifted relative to each other, with the nitrogen further separated from the metal at lower binding energies lonization of the metal adjacent nitrogen leads to more intense satellite structure than the ionization of the nitrogen atom further separated from the metal. This can be rationalized by one-electron considerations involving the HOMO and LUMO of the two equivalent-core-ton ground states The larger excitation energy of the satellites in cuordinated nitrogen systems with respect to coordinated $\mathrm{CO}$ can be traced back to strong contributions of doubly excited states due to the weaker bonding in $\mathrm{N}_{2}$ coordinated systems

(d) The spectrum of the finite complex shows all the characteristics of the photoelectron spectra of nitrogen adsorbed on transition-metal surfaces The analysis presented lends support to an interpretation of the core spectra of adsorbed nitrogen in terms of the ionization of two inequivalent nitrogen atoms rather than in terms of substrate band structure. It supports a "surface-molecule" concept for the interpretation of photoelectron spectra even for weakly chemisorbed molecules

\section{Acknowledgement}

We thank the "Regionales Rechenzentrum der Unıversitát zu Kòln" for computer time. We are indebted to Professors G. Hohlneicher and E.W. Plummer for many useful discussions, their interest in this work and for their support.

\section{References}

[1] J C. Fuggle E Umbach. D Menzel K Wandelt and C.R Brundle. Solid State Commun 27 (1978) 65

[2] J C Fuggle and D Menzel Vakuum Technik 27 (197S) 130

[3] O Gunnarsson and K Schonhammer, Phys Res. Letters 41 (1978) 1608

[4] EW Plummer W R Salaneck and J M Miller. Phys Rer B18 (1978) 1673
[5] H-J Freund and E W Plummer Phys Rex. B23 (1981) 4859.

[6] TN Rhodin and $G$ Ertl eds The nature of the surface chemucal bend (North-Holland Amsterdam 1979)

[7] E Unibach. Surface Sc1 117 (1982) 482

[S] R P Messmer SH Lamson and D R Salahub. Phys Rer $B 25$ (1982) 3576

[9] M Goltze. W Hirschusid is Grunze and R K Drincoll. to be published

[10] $K$ Horn. $I N$ DiNardo, W Eherhardt. $H-J$ Freuad and EW Plummer Surface So $115(1952) 465$

[11] PS Bagus, C R Brundle $K$ Hermann and D Vien. el. J Electron Spectry 20 (1980) 253

[12] E Umbach. A Sehichl and D Menzel Solıd State Commun $36(1950) 93$

[13] $Y$ Hsu K Jacohı and H H Rotermund Surface Sa 117 (1982) 581

[14] A B Anderson and R lloffmann J Chem Phys 61 (1974) 4545

[15] K Hermann PS Bagus CR Brundle and D Vienzel. Phys Rev B24 (19\$1) 7025

[16] D. Saddes H-J Frcund and G Hohlnescher. Surface $S c_{1}$ $85(1980) 527$

[17] A Schich]. D Menzel and A Rouch. Chem Phrs 65 (1982) 225

[18] H Itoh G Ertl and A B hunz. Chem Phys 59 (19S1) 149

[19] T A Taylor and PJ Estrup I I acuum Sa Technol 20 (1973) 26

H H V1adden J Kuppers and G Ertl $\mathrm{J}$ Chem Phrs $5 \mathrm{~s}$ (1973) 3401

[20] If Grunze. R K Driscoll. G V Burland J C L Gornish and I Pritchard Surface Sci 89 (1979) 381

[21] D Sellmann Angew Chem $\$ 6(1974) 692$

[22] G D Watt J Am Chem Soc 94(1972)7351

[23] U. Gelius J Electron Spectry 5 (1974) 985

[2-4] J.C Fuggle and D. Menzel in Proceedings of the 7th Internatuonal Vacuum Congrese and ird International Conference on Solsd Surfaces Vienn.z (1977) p 1003

[25] H D Polaschegg tppl. Phy 4 (1974) 63

[26] $\mathrm{H}-J$. Freund and $\mathrm{G}$ Hohineicher Theoret Chim Acta 51 (1979) 145

[27] H-J Freund. B Dich and G Hohine«her. Theoret Chim Acta 57 (1980) is]

[28] B Dich and $G$ Hohlneither Theorel Chm Alta 53 (1979) 221

[29] II L Jolly. in Electron speciroxcops. ed DA Shirlę (North-Holland. Amsierdam. 1972)

[30] $\mathrm{H}-J$ Freund and R W Bigelow. Chem Phys 55 (19SI) 407

[31] $T$ Aberg Ann Acad Sa Fenn Al 308 (1969) 7

[32] R. Munne and T Aberg Chen Phys Letters 7 (1970) 282.

[33] PS Bagus it Schrenk. DW Davis and D A Shirley Phys Rev 19 (1974) 1090

[39] P R. Norton R C Tapping and 1.W Goodale. Surface So1 72 (197S) 33 
[35] G H Leigh. J M Murrell. W. Bremser and W G Proctor. Chem Commun (1970) 1661.

[36] H Binder and D Sellmann, Angew. Chem 12 (1973) 1017

[37] P. Finn and W L Jolly, Inorg Chem 11 (1972) 1434

[38] B Folkesson, Acta Chem Scand 27 (1973) 287

[39] A Malek, B Folkesson and $R$ Larsson, Acta Chem Scand 34 (1980) 483
[40] W R Roduell, M F. Guest, T Darko, I H Hillier and J Kendrick, Chem Phys 22 (1977) 467.

[41] J Rose, T. Shubuya and V Mckoy, J Chern Phys 58 (1973) 74

[42] U. Gelius. Physica Scripta 9 (1974) 133

[43] H -J Freund. H. Pulm and B Dick. to be published

[44] L Brillounn. Act Sci Ind 71 (1943) 159 